\title{
LIQUID FLOW SENSOR FOR SMART IMPLANTABLE VP SHUNTS
}

\author{
Eniko T. Enikov ${ }^{1}$, Gergo Edes ${ }^{1}$, Rein Anton, ${ }^{2}$ and Jesse Skoch ${ }^{2}$ \\ ${ }^{1}$ University of Arizona, Advanced Micro- and Nanosystems Laboratory, USA \\ ${ }^{2}$ University of Arizona, Department of Surgery USA
}

\begin{abstract}
This paper describes the development of a novel liquid flow sensor based on the combined use of GMR magnetic sensors and micro-fabricated ferromagnetic sensing elements. A motivating application behind this effort is the development of a "smart" ventriculo-peritoneal (VP) shunt, capable of measuring the amount cerebral-spinal fluid drained from the ventricles of a person suffering from hydrocephalous. Unlike classical liquid flow sensors based on piezo-resistive flaps, the device presented here utilizes electroplated micro-fabricated ferromagnetic flaps (Ni), while the detector is an ultra-sensitive non-contact GMR sensor, separated from the working fluid by the lumen of the shunt.
\end{abstract}

\section{INTRODUCTION}

\section{Motivation}

Drainage of cerebral spinal fluid through shunting is a longterm treatment option for hydrocephalus, and is one of the most common neurosurgical procedures with an incidence rate of 5.5 per 100,000 [1]. The shunts are placed through the skull into the ventricle and drain the CSF into the peritoneal cavity as illustrated in Fig. 1a. Implanted cerebrospinal fluid shunts have been in use since 1950 s, however approximately $39 \%$ of shunts fail within the first year, 53\% fail within the first two years, and about $80 \%$ fail at some point after implantation [2]-[4]. The diagnosis and repair of failed VP shunts for hydrocephalus is one of the most common neurosurgical procedures, with over 27000 surgeries performed each year in the United States [5]. Previous efforts to develop smart VP shunts have included integrating pressure sensors for monitoring intra-cranial pressure [6] and development of thermal signature sensors (Shunt Check) to determine presence of flow based on the rate of re-heating of tissue [7]. Unfortunately, none of these methods resulted in a reliable flow sensing. Micro-flow sensors offer a direct method to measure flow and can be miniaturized to fit inside the lumen of a catheter. Existing approaches based on hot-wire anemometry, while small and sensitive, require continuous power in the range of 10-20 $\mathrm{mW}$ [8]. Alternatives based on peizo-resistive flap-style flow sensors require stringent encapsulation of the sensing elements to protect them against diffusion of sodium and other interfering ions [9]. The flow sensor presented here alleviates the packaging requirements of the sensor by separating the detector (an ultrasensitive GMR sensor) from the working fluid interacting with a micro-fabricated ferromagnetic flap as illustrated in Fig. 1b.

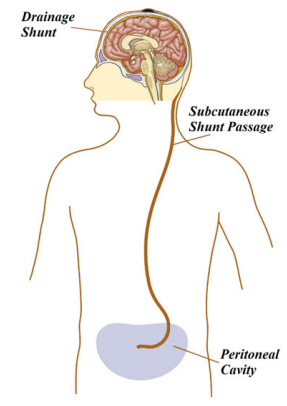

(a)

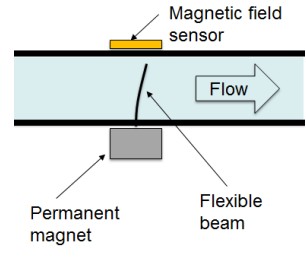

(b)
Figure 1: (a) Placement of VP shunt; (b) Flow detection using flexible ferromagnetic flap.

\section{Sensor Operating Principle}

The proposed flow sensing element is a ferromagnetic flap hinged about two attachment points to the substrate. Its magnetization is generated by a permanent magnet as shown in Fig. 2a. Under the action of viscous drag, the flap deflects laterally and alters the distribution of the magnetic field. These small changes are then detected by a GMR sensor placed adjacent to the elastic beam. An order-of-magnitude estimate of the drag force applied on the elastic beam by a slowly moving fluid can be obtained from the solution of Stokes flow past a prolate spheroid with major axis $a$ and minor axis $b(b<a)$ using the method of superposition of internal distributions of singularities [10]

$$
F_{d}=32 \pi \mu a v e^{3}\left[2 e+\left(3 e^{2}-1\right) \ln \frac{1+e}{1-e}\right]^{-1}
$$

where $\mu$ is the dynamic viscosity of CSF, and $v$ is its mean velocity, and $e=\sqrt{1-a^{2} / b^{2}}$ is the eccentricity of the prolate spheroid. In the case of water, with nominal flow rates of 20-30 $\mathrm{mL} / \mathrm{hr}$ in a channel with cross-section of $1.8 \mathrm{~mm} \times 5 \mathrm{~mm}, E q$. (1) predicts forces in the range of $40-60 \mu \mathrm{N}$. Detection of such forces is well within the capabilities of micro-cantilevers.

The following sections describe a more-accurate analysis and experimental validation of the operation of the proposed sensor. Signal-to-noise ratio analysis is provided in the last section of the paper along with suggestions for improvement of the design aimed at demonstrating a functional prototype of the envisioned smart VP shunt.

\section{SENSOR DESIGN}

\section{Mechanical Design}

Accurate prediction of the response of the transducing element to fluid flow requires fluid-structure interaction analysis. The drag force model represented by Eq.(1) neglects the effects of the channel walls and the shape of the transducing element In this work, a channel with a width of $2 \mathrm{~mm}$ and depth of $4 \mathrm{~mm}$ was chosen in order to meet the constraints of available machining tools while approaching the size restrictions of a future implantable device. The gap between the transducer and the channel wall was chosen to be $0.15 \mathrm{~mm}$, which turned out to be the lowest value allowing semi-manual assembly. The effect of this gap is critical to the sensitivity of the resulting flow sensors. The sensing element is a folded micro-cantilever anchored to the bottom of the channel shown in Fig. 2a.

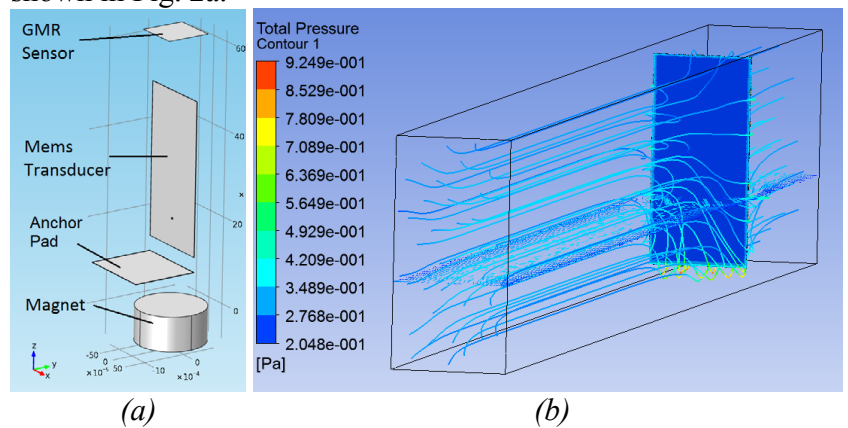

Figure 2: (a) Geometry for sensing element (left); and (b) The resulting flow pattern around it (right). 
A commercial software (CFX, Ansys Inc.) was used to carry out a two-step finite element analysis in order to optimize the size of the flap and the stiffness of the micro-cantilever hinges. The resulting flow and pressure distribution (see Fig 2b) was used to compute the deflection of the plate using a structural finite element model in ANSYS Mechanical (Ansys Inc.). Figure 3a shows the effect of changing the gap between the flap and the channel walls, while Figure $3 \mathrm{~b}$ shows the effect of changing the hinge length while maintaining a constant total length of the flap. It can be observed that reducing the gap from $150 \mu \mathrm{m}$ to $50 \mu \mathrm{m}$ doubles the deflection and hence the sensitivity. Furthermore, optimal hinge length results, since longer hinges lead to softer beam, but also increase the by-pass of the fluid around them. A maximum deflection of around $32 \mu \mathrm{m}$ microns is observed for a $20 \mathrm{~mL} / \mathrm{hr}$ flow rate.

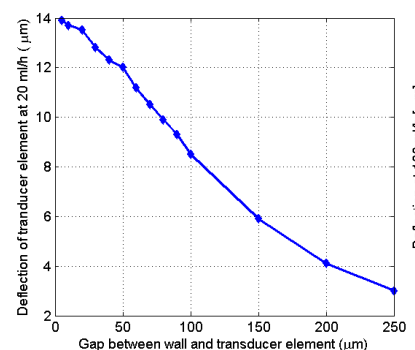

(a)

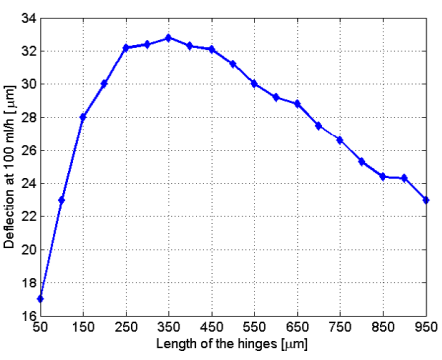

(b)
Figure 3: (a) Flap deflection vs. gap; and (b) Flap deflection vs. hinge length for a fixed total length of the transducer. Flow rate is $20 \mathrm{~mL} / \mathrm{hr}$.

\section{Magnetic Design}

The magnetic field analysis was carried out using commercial finite element code (COMSOL Inc.). The model includes an Alnico magnet (dimensions: $\phi 1.6 \mathrm{~mm} \times 0.8 \mathrm{~mm}$ ) with remnant magnetization of $0.75 \mathrm{~T}$. The transducer material was electroplated Ni with permeability of $\mu_{\mathrm{r}}=600$, while the GMR sensor was represented by a $1 \mathrm{~mm} \times 1 \mathrm{~mm}$ interrogation area above the flap. As anticipated, the simulation shows that the nickel flap is directing the magnetic field lines along its longitudinal axis and thus distorting the magnetic field around the GMR sensor when the transducer is deflected (see Figs. 4a-b). A series of combined electro-mechanical simulations representing flow rates between 5 and $100 \mathrm{~mL} / \mathrm{hr}$ have been carried out in order to produce a flow rate vs. magnetic field change plot (see Fig. 5). The resulting slope is near constant indicating a sensor sensitivity of $70 \mathrm{nT}-\mathrm{hr} / \mathrm{mL}$.

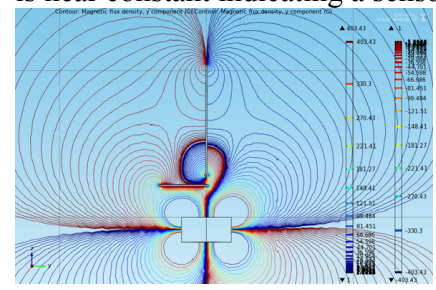

(a)

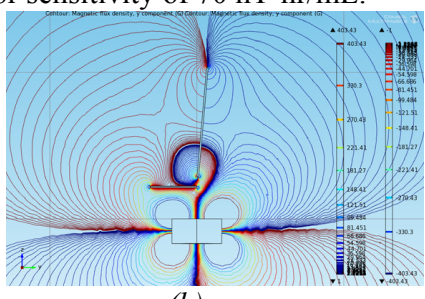

(b)
Figure 4: Contours of the magnetic flux density $B \_y$ around the transducer element: (a) no deflection; (b) after $5^{\circ}$ rotation.

\section{FABRICATION AND TESTING}

\section{Fabrication}

A Ni flap with lateral dimensions of $W=1.7 \mathrm{~mm}, L=350$ $\mu \mathrm{m}, H=3.9 \mathrm{~mm}$, and thickness of $10 \mu \mathrm{m}$ was fabricated via electro-plating into a photoresist mold over a sacrificial $\mathrm{Cu}$ seed layer. Release holes with diameter of $30 \mu \mathrm{m}$ were used to facilitate the release of the flap from the glass substrate. The fabrication sequence is summarized in Fig. 6. Briefly, $\mathrm{Cr}$ adhesion layer is evaporated onto a borosilicate glass wafer. A 2- $\mu$ m-thick $\mathrm{Cu}$ layer is then evaporated to serve as a sacrificial release layer. A $10-\mu \mathrm{m}-$ thick layer of photoresist was spun and patterned to produce a electroplating mold, followed by electro-deposition resulting in a $10-\mu \mathrm{m}$-thick layer of nickel. The substrate was diced into individual die, and subsequently, each die was etched individually in $\mathrm{Cu}$ and $\mathrm{Cr}$ etchants for 17 and 2 minutes, respectively. The release protocol is described earlier [11]. Powerful neodymium magnets were used to erect the transducers out of plane (see Fig. 6 (right)). The raised flaps were then assembled under an inverted Ushaped channel, whereby the glass substrate was used as the bottom wall of the channel (see Fig. 7-8).

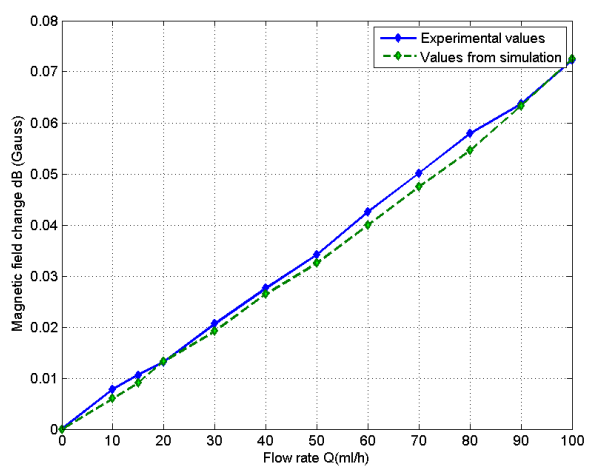

Figure 5: Magnetic field change vs. flow rate.

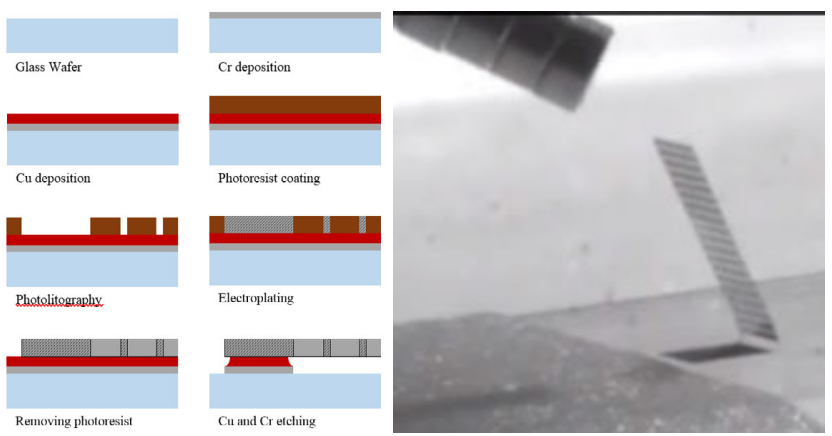

Figure 6: Fabrication sequence (left) and erecting the flap (right).
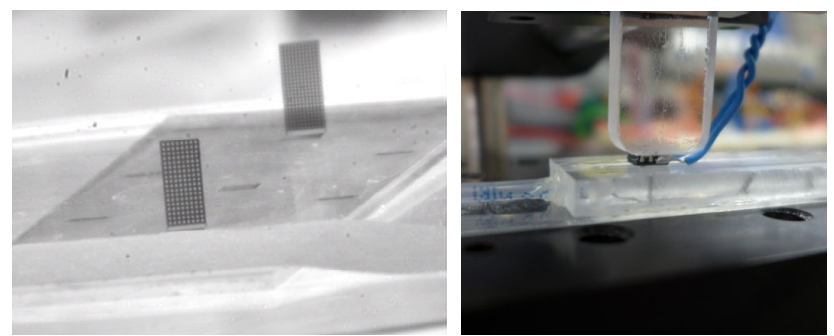

Figure 7: Released sensors prior to insertion in the flow channel (left) and assembled channel-sensor substrate with GMR sensor mounted superiorly (right).

\section{Experiments}

The magnetic field was measured with a Micromagnetics MTJ-240 magneto-resistive sensor placed above the channel was shown in Fig. 7. The manufacturer-supplied sensitivity of the sensor was $1 \% / \mathrm{G}$. The FE analysis described earlier was used to estimate the expected magnetic field change ranging between 0.006 and $0.07 \mathrm{G}$. This change corresponds to a resistance variation of 0.1 to $1 \Omega$ for a $1340-\Omega$ sensing resistor. A signal 
amplification circuit comprised of a Wheatstone bridge, a twostage amplifier with a total gain of 3300 , and a second-order lowpass filter with a cut-off frequency of $6 \mathrm{~Hz}$ was used to condition the sensor signal (see Fig. 9).

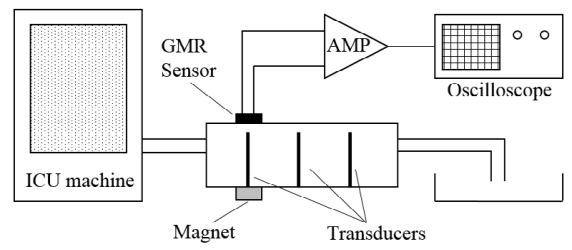

Figure 8: Test setup for flow measurements.

The output from the measurement circuit was connected to a Textronix TDS 3014B oscilloscope, and a Dataq DI-155 13-bit analog to digital converter. The flow rate was controlled by an Alaris PC 815 ICU/8110 syringe pump.

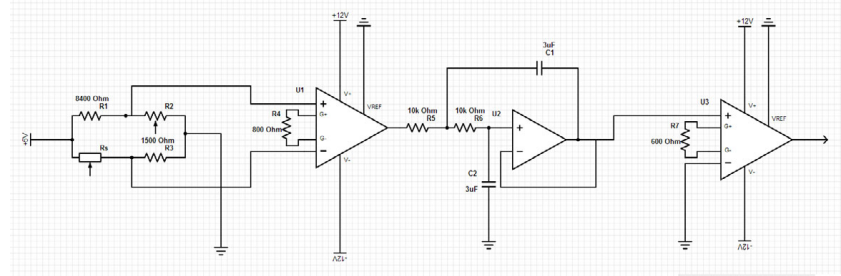

Figure 9: Sensor signal conditioning circuit.

The test procedure consisted of setting the flow rate to values between 10 and $100 \mathrm{~mL} / \mathrm{hr}$ in increments of $10 \mathrm{~mL} / \mathrm{hr}$. At least three interruptions of the flow were made at each flow setting, in order to examine the repeatability of the measurements. A sample data from the measurement taken at $50 \mathrm{~mL} / \mathrm{hr}$ is shown in Fig. 10.

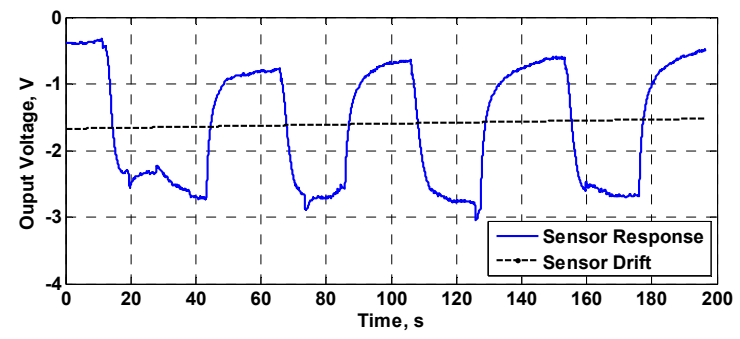

Figure 10: Sensor signal conditioning circuit.

Voltage changes between the on- and off-periods were recorded three times for each flow level to obtain a sensor calibration plot as shown in Fig. 11. Possible sources of the observed errors are the temperature drift of the sensor, environmental EM interference, and bubble formation around the transducer elements.

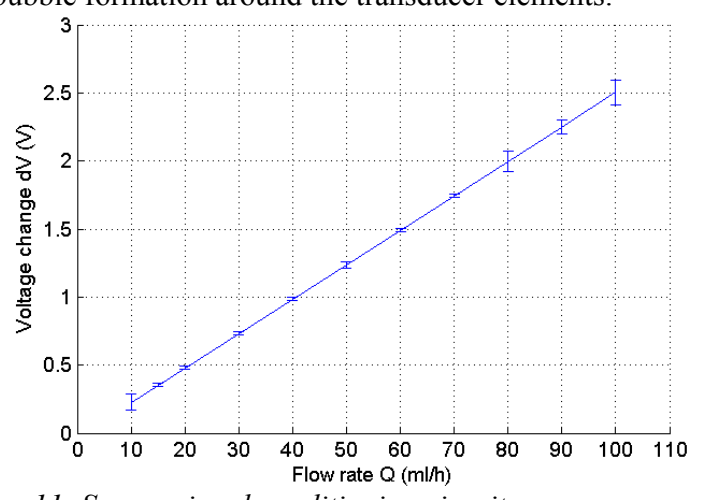

Figure 11: Sensor signal conditioning circuit.
To further analyze the response, the sensor output data were converted to an equivalent magnetic field changes according to

$$
\Delta B^{\exp }=\frac{\Delta R^{\exp }}{R_{S} S},
$$

where $S=1 \%$ is the manufacturer-supplied sensitivity of the GMR sensor and $R_{S}=1380 \Omega$ is the sensor resistance at zero magnetic field. The resistance change of the sensor for different flow rates was estimated using

$$
\Delta R^{\exp }=\frac{\Delta V^{\exp }\left(R_{S}+R_{3}\right)^{2}}{G V_{0} R_{S}},
$$

where $\mathrm{G}=3300$ is the amplification gain, $R_{3}=1500 \Omega$ is the value of the reference resistors of the Wheatstone bridge, and $V_{0}=5 \mathrm{~V}$ is the applied voltage. As demonstrated in Fig. 9, a near-constant sensitivity of $25 \mathrm{mV}-\mathrm{hr} / \mathrm{mL}$ has been observed over the entire range of interest. A low-frequency drift of $90 \mathrm{mV} / \mathrm{min}$ was also observed. The amplified and filtered signal had a noise level of 18 $\mathrm{mV}$-rms and a low-frequency drift of $90 \mathrm{mV}$ over a period of 1 minute.

\section{DISCUSSION AND CONCLUSIONS}

Analysis of the sensor output noise level implies a resolution of $1.4 \mathrm{~mL} / \mathrm{hr}$ and a drift of about $216 \mathrm{~mL} / \mathrm{hr}$. The observed lowfrequency drift and associated error over a 24-hr period is prohibitively high and needs to be reduced. Possible sources of the drift are fluctuations of the ambient magnetic field, low-frequency noise of the GMR sensor, and thermal noise (drift) due to the exposure of the sensor to the laboratory environment and selfheating. The Earth's ambient magnetic field is 25,000-65,000 nT. Its daily fluctuation however is typically $10-100 \mathrm{nT}$ [12], therefore, the associated drift would be around $1 \mathrm{~mL} / \mathrm{hr}$. Vehicular traffic and elevators could cause variations of 10-150 nT, which would imply an error of $2 \mathrm{~mL} / \mathrm{hr}$. GMR sensors are known to exhibit $1 / f$ low frequency noise [13]. Integrating the noise spectral density published in [13] over a period of one hour results I a noise level of $0.6 \mathrm{mV}$-rms. Equivalently, the corresponding a drift would be $0.03 \mathrm{~mL} / \mathrm{hr}$. Given the large difference between this predicted drift and observed one, it is concluded that the most likely source of the experimental drift are thermal fluctuations due to self-heating and thermo-resistive changes. The power dissipated in the sensor was approximately $8.3 \mathrm{~mW}$, which is high compared to the state-of-the art sensor readout schemes.

In summary, the development of a functional VP shunt based on GMR sensors will require cancellation of the Earth's ambient magnetic field, through the use of a second (reference) GMR sensor and reduction of the observed thermal drift. The later could be achieved by reduction of the excitation current and use of thermal compensation circuitry. The former is likely to require an increase in the signal to noise ratio, since the reduction of selfheating by two orders of magnitude would requires a 10 -fold reduction in the applied voltage, leading to a 10 -fold reduction in the gain of the system. It is therefore likely that an additional 10fold increase in the signal-to-noise ratio would be required. Such increase can be achieved by reducing the by-pass gap between the flap and the channel walls as demonstrated in Fig. 3a. Further increase in the sensitivity could come from reduction of the distance between the GMR sensor and the transducing flap. Analysis of the magnetic field distribution around the tip of the sensing element showed that for every $400 \mu \mathrm{m}$ of reduction of the distance between the GMR sensor and the flap, the signal doubles. Finally, the use of a stronger neodymium magnets or micromagnets, electroplated directly onto the flexible flap, is yet another 
approach to achieve the desired increase of signal-to-noise ratio.

\section{ACKNOWLEDGEMENTS}

The authors acknowledge the support for this work from the Rosztoczy Foundation through a grant to Gergo Edes and partial support from the National Science Foundation Grant \# 1353094

\section{REFERENCES}

[1] Y. Wu, N. L. Green, M. R. Wrensch, S. Zhao, and N. Gupta,"Ventriculoperitoneal shunt complications in California: 1990 to 2000," Neurosurgery, vol. 61, no. 3, (2007), pp. 557-563

[2] H.J. Garton, J.R. Kestle, and J.M. Drake, "Predicting shunt failure on the basis of clinical symptoms and signs in children," Journal of neurosurgery, vol. 94, no. 2, (2001) pp. 202-210,

[3] J.M. Drake, J R. Kestle, R. Milner, G. Cinalli, F. Boop, J. Piatt Jr, S. Haines, S. J. Schiff, D. D. Cochrane, P. Steinbok et al., "Randomized trial of cerebrospinal fluid shunt valve design in pediatric hydrocephalus," Neurosurgery, vol. 43, no. 2, (1998) pp. 294-303.

[4] J. Kestle, J. Drake, R. Milner, C. Sainte-Rose, G. Cinalli, F. Boop, J. Piatt, S. Haines, S. Schiff, D. Cochrane et al., "Longterm followup data from the shunt design trial," Pediatric neurosurgery, vol. 33, no. 5, (2001), pp. 230-236.

[5] K. Sheykholeslami and K. Kaga, "The otolithic organ as a receptor of vestibular hearing revealed by vestibular-evoked myogenic potentials in patients with inner ear anomalies," Hearing research, vol. 165, no. 1, (2002), pp. 62-67.

[6] V. Macellari, "Batteryless on-demand-sampling active radiosonde for intracranial pressure measurement," Medical and Biological Engineering and Computing, vol. 19, no. 6,(1981) pp. 686-694.
[7] J.R Madsen, G.S. Abazi, L. Fleming, M. Proctor, R. Grondin, S Magge, P. Casey, and T Anor. "Evaluation of the shuntcheck noninvasive thermal technique for shunt flow detection in hydrocephalic patients." Neurosurgery, v.68(1), (2011), pp.198-205.

[8] A. Glaninger, A. Jachimowicz, F. Kohl, R. Chabicovsky, and G. Urban, "Wide range semiconductor flow sensors," Sensors Actuat. A, 85, (2000), pp.139-146.

[9] J Chen, J Engel, C Liu, "Development of polymer-based artificial haircell using surface micromachining and 3D assembly", TRANSDUCERS, Solid-State Sensors, Actuators and Microsystems, 12th International Conference on, 2. IEEE, (2003).

[10] L.G. Leal, Advanced transport phenomena: fluid mechanics and convective transport processes. Cambridge University Press, vol. 7, 2007

[11] E.T. Enikov and K.V. Lazarov, "PCB-integrated metallic thermal microactuators," Sensors and Actuators A, vol. 105, no. 1, (2003), pp. 76-82.

[12] S. James and P. Spottiswoode, Effect of Ambient Magnetic Field Fluctuations on Performance in a Free Response Anomalous Cognition Task: A Pilot Study, Proceedings of the 36 th Annual Convention of the Parapsychological Association", (1993)

[13] M. Pannetier, C.Fermon, G. Le Goff, J. Simola, E. Kerr, J. Coey, "Noise in small magnetic systems - Applications to very sensitive magnetoresistive sensors", Journal of Magnetism and Magnetic Materials, v. 290-291, (2004), pp 1158-1160.

\section{CONTACT}

*ET. Enikov, Public tel: +1-520-621-4506; enikov@email.arizona.edu 